\title{
Characterization of the Serralysin-Like Gene of 'Candidatus Liberibacter solanacearum' Associated with Potato Zebra Chip Disease
}

\author{
Aravind Ravindran, Panatda Saenkham, Julien Levy, Cecilia Tamborindeguy, \\ Hong Lin, Dennis C. Gross, and Elizabeth Pierson ${ }^{\dagger}$
}

First and sixth authors: Department of Plant Pathology and Microbiology, second, third, and seventh authors: Department of Horticultural Sciences, and fourth author: Department of Entomology, Texas A\&M University, College Station 77843; and fifth author: Department of Agriculture-Agricultural Research Service, CDPG, San Joaquin Valley Agricultural Sciences Center, Parlier, CA 93648.

Accepted for publication 1 November 2017.

\begin{abstract}
The nonculturable bacterium 'Candidatus Liberibacter solanacearum' is the causative agent of zebra chip disease in potato. Computational analysis of the ' $C a$. L. solanacearum' genome revealed a serralysin-like gene based on conserved domains characteristic of genes encoding metalloprotease enzymes similar to serralysin. Serralysin and other serralysin family metalloprotease are typically characterized as virulence factors and are secreted by the type I secretion system (T1SS). The ' $\mathrm{Ca}$. L. solanacearum' serralysin-like gene is located next to and divergently transcribed from genes encoding a T1SS. Based on its relationship to the T1SS and the role of other serralysin family proteases in circumventing host antimicrobial defenses, it was speculated that a functional ' $\mathrm{Ca}$. L. solanacearum' serralysin-like protease could be a potent virulence factor. Gene expression analysis showed that, from weeks 2 to 6 , the expression of the ' $\mathrm{Ca}$. $\mathrm{L}$. solanacearum' serralysin-like gene was at least twofold higher than week 1 ,

indicating that gene expression stays high as the disease progresses. A previously constructed serralysin-deficient mutant of Serratia liquefaciens FK01, an endophyte associated with insects, as well as an Escherichia coli lacking serralysin production were used as surrogates for expression analysis of the ' $\mathrm{Ca}$. L. solanacearum' serralysin-like gene. The LsoA and LsoB proteins were expressed as both intact proteins and chimeric S. liquefaciens' $C a$. L. solanacearum' serralysin-like proteins to facilitate secretion in the S. liquefaciens surrogate and as intact proteins or as a truncated LsoB protein containing just the putative catalytic domains in the E. coli surrogate. None of the ' $\mathrm{Ca}$. L. solanacearum' protein constructs expressed in either surrogate demonstrated proteolytic activity in skim milk or zymogram assays, or in colorimetric assays using purified protein, suggesting that the ' $\mathrm{Ca}$. L. solanacearum' serralysin-like gene does not encode a functional protease, or at least not in our surrogate systems.
\end{abstract}

'Candidatus Liberibacter' spp. are nonculturable, gram-negative, phloem-limited bacteria associated with economically important diseases of potato, citrus, and many other crops (Gottwald 2010; Haapalainen 2014; Liefting et al. 2009; Munyaneza et al. 2010). 'Candidatus Liberibacter solanacearum' was confirmed as the causative agent of zebra chip (ZC) disease on potato based on graft transmission (Secor et al. 2009), and evidence indicates that ' $\mathrm{Ca}$. Liberibacter' spp. cause other diseases (e.g., 'Ca. L. asiaticus'associated citrus huanglongbing). $\mathrm{ZC}$ is an important disease of potato in the United States and many countries throughout the Americas and in New Zealand. ' $C a$. L. solanacearum' is vectored to potato by the tomato or potato psyllid (Bactericera cockerelli Sulc) (Liefting et al. 2009; Lin et al. 2011; Munyaneza et al. 2007; Secor et al. 2009; Wen et al. 2009). Characteristic symptoms of ZC include leaf chlorosis and purpling, stunted plants with swollen internodes, proliferation of axillary buds and aerial tubers, and plant decline leading to death (Munyaneza et al. 2007). Depending on the seasonal timing of infection, ZC can dramatically reduce tuber yields and typically causes a brown defect in the tubers, rendering them unmarketable. ZC affects all market classes of potato by reducing tuber yield and quality, and all cultivated potato varieties are susceptible to the disease (Diaz-Montano et al. 2014; Lévy et al.

${ }^{\dagger}$ Corresponding author: E. Pierson; E-mail: eapierson@tamu.edu

This work was supported by Texas A\&M AgriLife Research Zebra Chip Disease Program, Grant Number ZC 06-407135.

*The $\boldsymbol{e}$-Xtra logo stands for "electronic extra" and indicates that two supplementary figures are published online.

(c) 2018 The American Phytopathological Society
2015). Factors that contribute to ' $C a$. L. solanacearum' virulence are largely unknown.

Based on the absence of both the type III and type II secretion systems (T3SS and T2SS, respectively) in the ' $\mathrm{Ca}$. L. solanacearum' genome, Lin et al. (2011) postulated that the type I secretion system (T1SS) is the primary mechanism for the delivery of bacterial products important for ' $C a$. L. solanacearum' interactions with the host plant. In a search for potential virulence factors, computational analysis of the ' $\mathrm{Ca}$. L. asiaticus' genome resulted in the identification of a gene encoding a serralysin-family protein (CLIBASIA_01345) located next to a T1SS gene cluster (Cong et al. 2012). Moreover, comparative genomics revealed that serralysin-like genes are widely distributed in ' $\mathrm{Ca}$. Liberibacter' genomes (Cong et al. 2012). Wang and Trivedi (2013) reported that the ' $\mathrm{Ca}$. L. asiaticus' gene is highly expressed in citrus phloem, speculating that it may be important in ' $\mathrm{Ca}$. L. asiaticus' virulence.

Serralysin, first described from Serratia spp., is an approximately $50-\mathrm{kDa}$ metalloprotease (EC 3.4.24.40) originally isolated from the digestive system of silkworm (Miyata et al. 1971). Proteins in the serralysin family are characterized as bacterial extracellular metalloproteases having a highly conserved zinc-binding motif (HEXXHXUGUXH), a Met-turn (SXMXY) sequence, and multiple calcium-binding glycine-rich repeats (GGXGXD). Kaibara et al. (2012) reported that Serratia liquefaciens FK01, an endophytic species associated with insects, produced a serralysin with proteolytic activity that contributed to its ability to parasitize insects. Metalloproteases resembling serralysin are produced by several types of gram-negative bacteria, including many Serratia and Pseudomonas spp., Dickeya chrysanthemi, and Proteus mirabilis (Baumann 2011; Maeda 1996). In all cases, translocation occurs through a type I secretion channel and, in most of these reports, the serralysin functions in virulence. For example, a serralysin metalloprotease was 
identified as a factor that inhibited adhesion of immune cells and bacterial clearance, serving as a virulence factor in $S$. marcescens infection of silkworm (Bombyx mori) (Ishii et al. 2014). In plants, the production of antimicrobial proteins and peptides is a major component of plant defense responses, and metalloproteases have been shown to be important bacterial virulence factors (Castro and Fontes 2005). Thus, hypothetically, a functional serralysin family metalloprotease could be a potent, conserved ' $\mathrm{Ca}$. Liberibacter' virulence factor in plants.

Analyses of the genomes of both ' $\mathrm{Ca}$. L. solanacearum' haplotypes LsoA and LsoB that cause ZC (strains HenneA and ZC1, respectively) revealed the presence of a gene encoding a serralysinlike protein located next to a gene cluster encoding a T1SS (Lin et al. 2011). In this study, we report on the similarities between the ' $\mathrm{Ca}$. L. solanacearum' serralysin-like gene and other genes in the serralysin family in terms of the conserved motifs required for proteolytic activity. In order to examine the functionality of the protein encoded by this gene, a previously constructed serralysin-defective mutant of S. liquefaciens FK01 (Kaibara et al. 2012) was used as a surrogate for protein expression studies. The $S$. liquefaciens wild-type strain FK01 produces two serralysin-like metalloproteases, ser 1 and ser 2 , and serralysin-deficient mutants were generated by deletion of one or both of the genes (Kaibara et al. 2012). The mutants demonstrated loss of proteolytic activity in assays using skim milk agar and casein zymography, and genetic complementation restored proteolytic activity (Kaibara et al. 2012). Consequently, the $S$. liquefaciens FK01 double mutant S. liquefaciens FK04 was selected as a surrogate for examining whether the ' $\mathrm{Ca}$. L. solanacearum' serralysin-like gene encodes a functional protease. An Escherichia coli strain (DE3), which does not produce serralysin, also was used as a surrogate.

\section{MATERIALS AND METHODS}

Bacterial strains, growth conditions, plasmids, and primers. Populations of the potato or tomato psyllid vector Bactericera cockerelli (Central) infected with either ' $\mathrm{Ca}$. L. solanacearum' haplotypes LsoA or LsoB from separate colonies described previously (Nachappa et al. 2011) were provided by Dr. Cecilia Tamborindeguy. Total DNA (insect and bacterial) was isolated from infected psyllids using the method of Lévy et al. (2011). Initial screening of ' $C a$. L. solanacearum' in psyllids used the ' $C a$. L. solanacearum' TX 16/23 F/Rspecific primers described by Ravindran et al. (2011). In all infected psyllids used in this study to amplify the ' $\mathrm{Ca}$. L. solanacearum' serralysin-like gene, a single visible band 383 bp in length was obtained, confirming that each psyllid carried ' $\mathrm{Ca}$. L. solanacearum'. The ' $\mathrm{Ca}$. L. solanacearum' haplotype carried by each insect was confirmed using haplotype-specific primers, as described by Wen et al. (2013).

The bacterial strains and plasmids are listed in Table 1 and primers in Table 2. The $S$. liquefaciens strains were provided by Dr. K. Iiyama (Kaibara et al. 2012). The S. liquefaciens and E. coli $\mathrm{DH} 5 \alpha$ liquid cultures were grown in a Luria-Bertani (LB) medium at $28^{\circ} \mathrm{C}$ and supplemented with ampicillin or carbenicillin (100 $\mu \mathrm{g} \mathrm{ml}^{-1}$ ). The plasmid pBBR1 MCS-4 was provided by Dr. M. Kovach (Kovach et al. 1995).

TABLE 1. Bacterial strains and plasmid details

\begin{tabular}{|c|c|c|}
\hline Plasmids and strains & Description $^{\mathrm{a}}$ & Source \\
\hline \multicolumn{3}{|l|}{ Plasmids } \\
\hline pBBR1-MCS4 & Broad-host range plasmid cloning vector, $\mathrm{Amp}^{\mathrm{R}}\left(\mathrm{Car}^{\mathrm{R}}\right.$ or $\left.\mathrm{Pip}^{\mathrm{R}}\right)$ & Kovach et al. (1995) \\
\hline pET-14b & Escherichia coli expression vector (His-Tag), Amp $\left(\mathrm{Car}^{\mathrm{R}}\right.$ or $\left.\mathrm{Pip}^{\mathrm{R}}\right)$ & Novagen, Madison, WI \\
\hline \multicolumn{3}{|l|}{ Bacterial strains } \\
\hline Serratia liquefaciens FK01 & Wild-type strain & Kaibara et al. (2012) \\
\hline S. liquefaciens $\mathrm{FK} 04$ & ser1/ser2-double mutant of FK01 & Kaibara et al. (2012) \\
\hline E. coli $\mathrm{DH} 5 \alpha$ & Competent cells & ThermoFisher Scientific \\
\hline E. coli BL21 (DE3) & Competent cells & ThermoFisher Scientific \\
\hline \multicolumn{3}{|l|}{ S. liquefaciens $\mathrm{FK} 04$} \\
\hline pBBR1 S1 ser2 & 1,979 bp, EcoRI/BamHI, Car ${ }^{\mathrm{R}}$ & This study \\
\hline pBBR1 LsoA ser & 1,983 bp, EcoRI/BamHI, Car ${ }^{\mathrm{R}}$ & This study \\
\hline pBBR1 LsoB ser & 1,479 bp, EcoRI/BamHI, CarR & This study \\
\hline pBBR1 chimeric ser1-LsoA & 3,140 bp, XhoI/PstI, Car ${ }^{\mathrm{R}}$ & This study \\
\hline pBBR1 chimeric ser1-LsoB & 2,652 bp, XhoI/PstI, Car ${ }^{\mathrm{R}}$ & This study \\
\hline pBBR1 chimeric ser2-LsoA & 2,754 bp, EcoRI/BamHI, CarR & This study \\
\hline pBBR1 chimeric ser2-LsoB & 2,266 bp, EcoRI/BamHI, CarR & This study \\
\hline \multicolumn{3}{|l|}{ E. coli BL21 (DE3) } \\
\hline pET14b LsoB ser & 1,535 bp, XhoI/BamHI, Car ${ }^{\mathrm{R}}$ & This study \\
\hline pET14b LsoA ser & 2,001 bp, XhoI/BamHI, Car ${ }^{\mathrm{R}}$ & This study \\
\hline pET14b LsoB ser-300 & 1,238 bp, XhoI/BamHI, Car ${ }^{\mathrm{R}}$ & This study \\
\hline
\end{tabular}

${ }^{a} \mathrm{Amp}^{\mathrm{R}}, \mathrm{Car}^{\mathrm{R}}$, and $\mathrm{Pip}^{\mathrm{R}}$ indicate resistant to ampicillin, carbenicillin, and piperacillin, respectively.

TABLE 2. Primer details ${ }^{\mathrm{a}}$

\begin{tabular}{|c|c|c|}
\hline Primers & $5^{\prime}-3^{\prime}$ & Source \\
\hline Lso-ser qPCR F & ATCTTGGTCTAGTATTGGTGG & This study \\
\hline Lso-ser qPCR R & CCATTACATCCCATCCATCAG & This study \\
\hline Lso-ser F EcoRI & CACCTTgaattcTAGCGGAAGGAGATTCTTA & This study \\
\hline Lso-ser R BamHI & ATTCGTggatccGCAAACGAGTACAACAATAGCC & This study \\
\hline ser2 F EcoRI & CACCTTgaattcGAGAGCAGCGTCAGAACC & Kaibara et al. (2012) \\
\hline ser2 R BamHI & ATTTCCggatccGTAATAGAGCGGTGGGCATA & Kaibara et al. (2012) \\
\hline ProF (-811) XhoI & ACAActcgagGGATCCCCATGATCGTCAG & Kaibara et al. (2012) \\
\hline ser1-N R & AATTTCCATGTCTTCGCTGGCCGGGTGTTTCACG & This study \\
\hline Lso ser1-C F & GCGAAGACATGGAAATTAACCTAGATGCGAGAGATG & This study \\
\hline ser2-N R & AATTTCCATGTTGCCCAAGTTCAGGGTGCAA & This study \\
\hline Lso ser2-C F & TGGGCAACATGGAAATTAACCTAGATGCGAGAGATG & This study \\
\hline Lso-C ser R PstI & ATTCGTctgcagGCAAACGAGTACAACAATAGCC & This study \\
\hline Lso-ser F1 XhoI & CACCTTctcgagGTGGATGATATCAAGCCTGTG & This study \\
\hline Lso-ser R1 BamHI & TGCGggatccTCATTCACTAAAATCAAG & This study \\
\hline LsoB-ser-300 F XhoI & CACCTTctcgagATGGAAATTAACCTAGATGCGAGAGATG & This study \\
\hline
\end{tabular}

${ }^{a}$ Sequences are underlined with solid lines and dotted lines are complementary. 
Analysis of the serralysin-like gene in ' $\mathrm{Ca}$. L. solanacearum' and ' $\boldsymbol{C a}$. L. asiaticus'. The genome sequences for strains representing the two different ' $C a$. L. solanacearum' haplotypes LsoA and LsoB (HenneA and ZC1, respectively) are available at the National Center for Biotechnology Information (NCBI) for analysis. Comparison of serralysin-like genes among different ' $\mathrm{Ca}$. Liberibacter' spp. was performed using IMG gene content analysis (https://img.jgi.doe.gov/cgi-bin/w/main.cgi). To compare the serralysin gene of Serratia spp. with the serralysin-like gene of ' $\mathrm{Ca}$. Liberibacter' spp., the CLUSTAL X Multiple Sequence Alignment program was used (Thompson et al. 1997). Protein sequences used in the analysis are available in the NCBI database and included the following sequences: Ser1 (GenBank accession BAK39731) and Ser2 (GenBank accession BAK39733) from S. liquefaciens FK01, serralysin (GenBank accession WP_004934318) from $S$. marcescens, the serralysin-like proteins from ' $\mathrm{C} a$. L. solanacearum' ZC1 (haplotype LsoB) (GenBank accession WP_013461860) and HenneA (haplotype LsoA) (GenBank accession KJZ82330.1), and the serralysin-like protein from ' $\mathrm{Ca}$. L. asiaticus' strains gxpsy (GenBank accession WP_015452346) and psy62 (GenBank accession WP_015452346).

Expression of the serralysin-like gene by quantitative polymerase chain reaction assays. Quantitative real-time reversetranscription polymerase chain reaction (qRT-PCR) analysis was used to determine the level of expression of the ' $\mathrm{Ca}$. L. solanacearum' serralysin-like gene in tomato plants that had been infested with bacterialiferous psyllids. For this experiment, insects carrying LsoB were used. Total RNA was extracted using an RNeasy Mini Kit (Qiagen) from leaves of plants at week 1 (when insects were removed from plants) and weeks 2, 3, 4, and 6 after insect infestation. As a negative control at each time point, RNA also was extracted from plants treated with psyllids without ' $\mathrm{Ca}$. L. solanacearum'. RNA samples were treated with TURBO DNase (Ambion) according to the manufacturer's protocol. Total RNA samples were quantified using spectrophotometry (Nano-Drop Technologies, Inc.). Total RNA was reverse-transcribed into cDNA using a Super Script Vilo cDNA Synthesis kit (Invitrogen) following the manufacturer's protocol. The cDNA was quantified using spectrophotometry and samples were diluted to $10 \mathrm{ng} \mu \mathrm{l}^{-1}$. qRT-PCR primers were designed for the serralysin-like gene and the recA housekeeping genes of ' $\mathrm{Ca}$. L. solanacearum'. The following reaction mix was used for each 20- $\mu$ l qRT-PCR mixture: $10 \mu$ of SYBR GreenER qPCR SuperMix Universal, $8.16 \mu \mathrm{l}$ of nuclease-free water, $0.04 \mu \mathrm{l}$ of ROX reference dye, $0.4 \mu$ l of Lso-ser qPCR F primer ( $200 \mathrm{nM}$ final), $0.4 \mu \mathrm{l}$ of Lso-ser qPCR R primer ( $200 \mathrm{nM}$ final), and $1 \mu \mathrm{l}$ of template DNA (10 ng $\mu \mathrm{l}^{-1}$ ). qRT-PCR was performed on an Applied Biosystems 7500 Fast Real-Time PCR System. Data were analyzed using the comparative cycle threshold $(\mathrm{Ct})$ method, in which all $\mathrm{Ct}$ values were normalized to the $\operatorname{rec} A$ housekeeping gene. The $2^{-} \Delta \Delta \mathrm{Ct}$

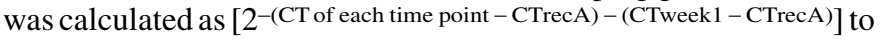
determine the relative expression of the ' $\mathrm{Ca}$. L. solanacearum' serralysin-like gene in weeks $2,3,4$, and 6 compared with week 1 .

Cloning of the ' $\mathrm{Ca}$. L. solanacearum' serralysin-like gene and S. liquefaciens serralysin. A single ' $\mathrm{Ca}$. L. solanacearum' primer set (Lso-ser F/R) was designed based on the conserved regions of the serralysin-like gene sequence (including the native ribosomebinding site) in both haplotypes as determined from analysis of the HenneA and ZC1 genomes. The serralysin-like genes of both haplotypes were amplified from the genomic DNA of ' $\mathrm{Ca}$. L. solanacearum'-infected psyllids having either LsoA or LsoB. A fragment containing the complete sequence of the ser 2 gene was amplified from genomic DNA of $S$. liquefaciens FK01 by PCR using primer pair ser2F/ser2R (Kaibara et al. 2012). All three amplified fragments were cloned separately into the EcoRI-BamH1 site in the multiple cloning locus of pBBR1 MCS-4, then transformed into E. coli $\mathrm{DH} 5 \alpha$. The presence of each insert was confirmed using colony PCR and verified by sequencing. The confirmed plasmids (pBBR1 LsoA ser, pBBR1 LsoB ser, and pBBR1 S1 ser2) were transformed separately via electroporation into $S$. liquefaciens FK04 (ser1/ser2 double mutant) and E. coli DE3 (does not make serralysin) to test for protease activity.

Screening for protease activity. Skim milk agar plate assays were used to detect proteolytic activity after incubation at $28^{\circ} \mathrm{C}$ for 5 days. Skim milk agar contained $10 \%$ skim milk powder (Oxoid; ThermoFisher Scientific), $0.5 \%$ peptone, and $1.5 \%$ agar (autoclaved for $5 \mathrm{~min}$ ). The formation of clear zones around colonies was diagnostic of proteolytic activity. For the zymography proteolytic assays, $20 \mu$ of cell culture was mixed with a $2 \times$ zymogram sample buffer (Bio-Rad) incubated at $20^{\circ} \mathrm{C}$ for $1 \mathrm{~h}$. A $20-\mu$ portion of the denatured samples was then separated by electrophoresis on a $10 \%$ polyacrylamide gel containing $0.05 \%$ casein with a $10 \mathrm{~mA}$ constant current (maximum voltage was $100 \mathrm{~V}$ ) at room temperature until the blue tracking dye reached the bottom of the gel. The gel was incubated in a renaturing buffer $(2.5 \%$ Triton X-100, vol/vol) for $30 \mathrm{~min}$, repeated a second time, and then incubated in zymogram developing buffer for $16 \mathrm{~h}$ with gentle agitation at $37^{\circ} \mathrm{C}$. The gel was stained with Coomassie Brilliant Blue R-250 and then destained (Kaibara et al. 2012). Areas of digestion appearing as clear bands against the stained background indicated protease activity.

Construction of chimeric Serratia- ' $\mathrm{Ca}$. L. solanacearum' serralysin-like genes. To circumvent possible problems with protein secretion in our surrogate systems, a two-step PCR method was used to make chimeric serralysin-like genes composed of all combinations of the $5^{\prime}$ sequence from the S. liquefaciens serl or ser 2 serralysin genes and the $3^{\prime}$ sequence encoding the putative catalytic regions from the LsoA or LsoB serralysin-like genes. The first step was to PCR amplify both of the 5' serralysin sequences from genomic DNA of $S$. liquefaciens FK01 (using forward primers ProF [-811] and ser2F and the reverse primers ser1-N R and ser2-N $\mathrm{R}$ for serl and ser 2 amplification, respectively), and both of the $3^{\prime}$ ' $C a$. L. solanacearum' sequences from the genomic DNA samples of psyllids carrying either LsoA or LsoB (using forward primers Lso-C ser1F and Lso-C ser2F and the reverse primer Lso ser-C R). The next step used the $5^{\prime}$ and $3^{\prime}$ amplicons as templates to join them by PCR amplification to form the four different, complete chimeric serralysinlike genes. This was made possible because the $5^{\prime}$ sequence reverse primers (ser1-N R and ser2-N R) and 3' sequence forward primers (Lso-C ser1F and Lso-C ser2F) carry the overlap sequence required to join the PCR products to form a chimeric sequence. The second PCR was performed using either the serl or ser2 forward primer (ProF [-811] and ser2F) and the ' $\mathrm{Ca}$. L. solanacearum' reverse primer (Lso ser-C R) (Supplementary Fig. S1). The chimeric amplicons were cloned into pBBR1 MCS-4 (Kovach et al. 1995), resulting in plasmids pBBR1 serl-LsoA, pBBR1 serl-LsoB, pBBR1 ser2-LsoA, and $\mathrm{pBBR} 1$ ser2-LsoB. The plasmids were transferred separately into E. coli $\mathrm{DH} 5 \alpha$ and colony PCR confirmed the presence and expected size of each chimeric Serratia- 'C $a$. L. solanacearum' serralysinlike gene. From there, sequences were transferred separately into S. liquifaciens FK04 and tested as above for proteolytic activity.

Proteolytic activity of the complete LsoA and LsoB serralysin-like proteins and a truncated LsoB protein. The complete LsoA and LsoB serralysin-like genes were amplified using the LsoA ser primer set (Lso-ser F1/Lso-ser R1) and LsoB ser primer set (Lso-ser F1/Lso-ser R), respectively, and then inserted separately into the XhoI-BamH1 site within the multiple cloning locus of the high-copy protein expression vector $\mathrm{pET} 14 \mathrm{~b}$ (Novagen), resulting in pET14b LsoA ser and pET14b LsoB ser, respectively. Because the 300 -bp region at the $\mathrm{N}$-terminal end of the LsoB serralysin-like gene does not contain the domains hypothetically involved in catalytic activity, a truncated construct containing just the putative catalytic domains was constructed (e.g., without other parts of the protein that potentially could negatively affect expression, secretion, or activity in the E. coli DE3 surrogate). For the truncated construct, the 300-bp region at the N-terminal end of the LsoB serralysin-like gene was excluded. The remaining 1,238bp sequence was amplified using forward and reverse primers 
LsoB-ser-300 F and Lso-ser R and was cloned into pET14b, resulting in plasmid pET14b LsoB ser-300. All plasmids were verified by sequencing and then transformed into E. coli DE3.

DE3 cells harboring pET14b LsoA ser, pET14b LsoB ser, or pET14b LsoB ser- 300 were grown overnight at $37^{\circ} \mathrm{C}$ in $3 \mathrm{ml}$ of LB supplemented with ampicillin $(100 \mu \mathrm{g} / \mathrm{ml})$. A 1:100 dilution of an overnight cell culture was added to $100 \mathrm{ml}$ of LB supplemented with ampicillin $(10 \mu \mathrm{g} / \mathrm{ml})$, grown to an optical density at $600 \mathrm{~nm}$ of 0.4 to 0.5 , then induced with $1 \mathrm{mM}$ isopropyl- $\beta$-D-thiogalactoside (IPTG) for $3 \mathrm{~h}$. After induction with IPTG, cells were harvested, resuspended in cell lysis buffer A (20 mM phosphate buffer [pH 7.8] containing $0.5 \mathrm{M} \mathrm{NaCl}$ and $8 \mathrm{M}$ urea), and lysed via sonication. The protein expression was checked by sodium dodecyl sulfate polyacrylamide gel electrophoresis (SDS-PAGE) analysis, and proteolytic activity was evaluated using the zymogram assay (as above). The LsoB and LsoB ser-300 proteins were purified using affinity chromatography (Ni-NTA column). The column was washed with buffer B (20 mM phosphate buffer [pH 7.8] containing $10 \mathrm{mM}$ imidazole, $0.5 \mathrm{M} \mathrm{NaCl}$, and $8 \mathrm{M}$ urea) and the protein was eluted with buffer $\mathrm{C}\left(20 \mathrm{mM} \mathrm{NaH}_{2} \mathrm{PO}_{4}\right.$ [pH 4.0], $0.5 \mathrm{M} \mathrm{NaCl}$, and $8 \mathrm{M}$ urea). The purified protein was transferred to a dialysis bag to remove the urea; the buffer used contained a urea gradient $(4 \mathrm{M}, 2 \mathrm{M}, 1 \mathrm{M}, 0.5 \mathrm{M}$, and
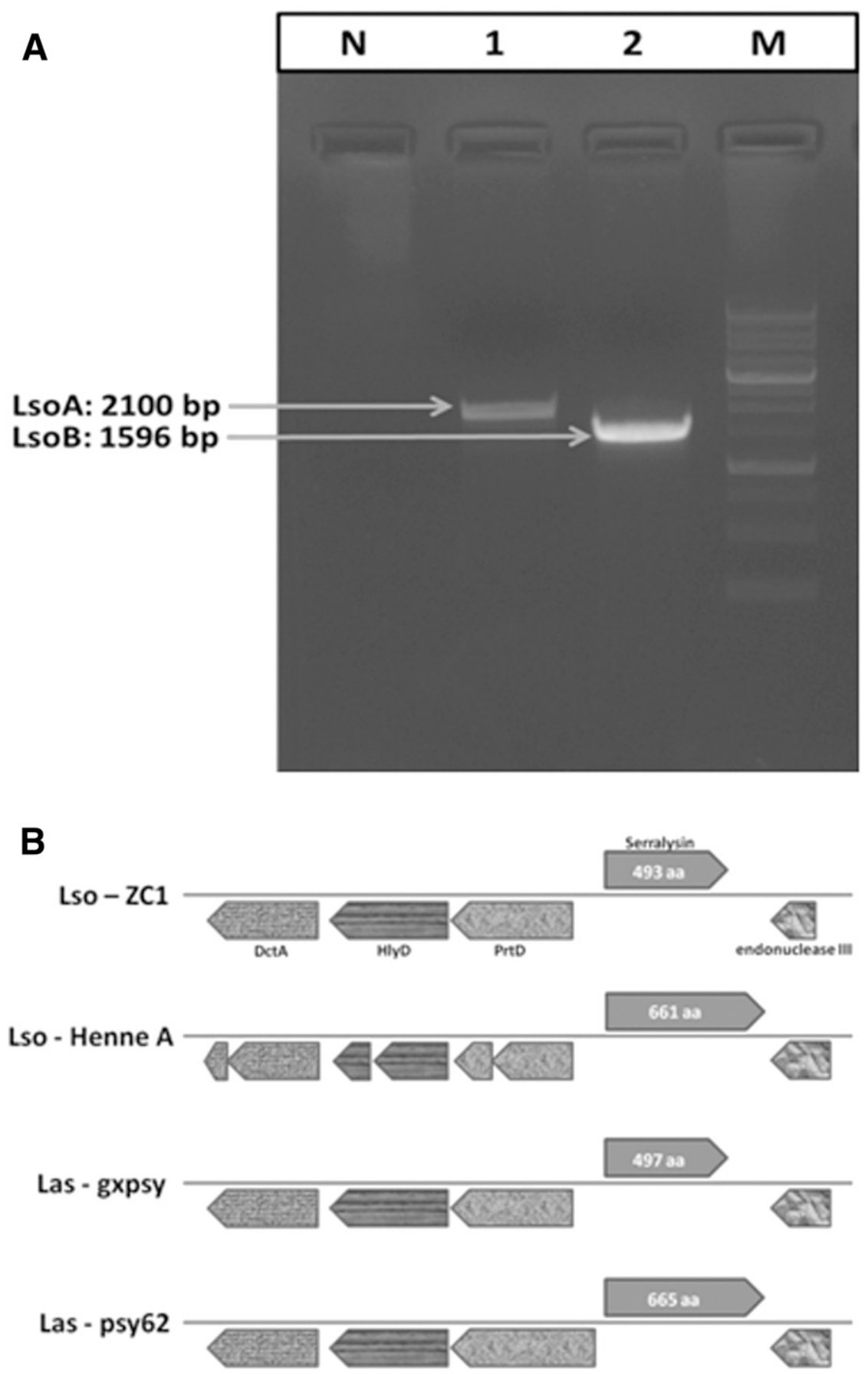

Fig. 1. Serralysin-like genes from 'Candidatus Liberibacter solanacearum' haplotypes LsoA and LsoB. A, Polymerase chain reaction amplification of the 'Ca. L. solanacearum' serralysin-like gene from total genomic DNA obtained from psyllids carrying either the LsoA or LsoB haplotype. Lane N, water control; lane 1, LsoA; lane 2, LsoB; lane M, molecular size marker (1-kb DNA ladder, 250 to 10,000 bp). B, Comparison of orthologs of the serralysin-like gene cluster of ' $\mathrm{Ca}$. L. solanacearum' strain ZC1 (LsoB haplotype), 'Ca. L. solanacearum' strain HenneA (LsoA haplotype), 'Ca. L. asiaticus' strain gxpsy, and 'Ca. L. asiaticus' strain psy62 for similar orthologs. Shading indicates ortholog groups and the size of the bar approximately corresponds to gene size. This comparison was performed using JGI-IMG/ER (https://img.jgi.doe.gov/cgi-bin/w/main.cgi). Upstream genes of serralysin are a type I secretion ATPase (PrtD), a type I secretion membrane fusion protein (HylD), and a $\mathrm{C}_{4}$-dicarboxylate transporter (DctA). 
$0 \mathrm{M})$. The protein purity was checked by SDS-PAGE analysis, and proteolytic activity was evaluated using the zymogram assay and a colorimetric assay. For the colorimetric assay, azocasein was used as a substrate with the purified proteins, as described by (Secades and Guijarro 1999). Enzyme solutions $(120 \mu \mathrm{l})$ were mixed with $480 \mu \mathrm{l}$ of $1 \%$ azocasein (Sigma-Aldrich) in Tris buffer containing $\mathrm{MgCl}_{2}$, and the mixtures were then incubated at $30^{\circ} \mathrm{C}$ for $30 \mathrm{~min}$. To precipitate nondigested azocasein, $600 \mu \mathrm{l}$ of $10 \%$ trichloroacetic acid (TCA) was added and incubated on ice for $30 \mathrm{~min}$, after which the reactions were centrifuged $\left(15,000 \times g\right.$ at $4^{\circ} \mathrm{C}$ for $\left.10 \mathrm{~min}\right)$. Supernatants $(800 \mu \mathrm{l})$ were neutralized by adding $200 \mu \mathrm{l}$ of $1.8 \mathrm{~N} \mathrm{NaOH}$. The absorbance of supernatants was measured using a spectrophotometer $(415 \mathrm{~nm})$, where increased absorbance relative to the control indicated the presence of proteolytic activity.

\section{RESULTS}

Analysis of the serralysin-like genes in ' $\mathrm{Ca}$. L. solanacearum' and ' $\boldsymbol{C} \boldsymbol{a}$. L. asiaticus'. The ' $\mathrm{Ca}$. L. solanacearum' serralysin-like gene sequences for haplotypes LsoA and LsoB were identified from the genomic sequences of ' $\mathrm{Ca}$. L. solanacearum' strains HenneA and ZC1, respectively, in the NCBI database. The BlastP analysis identified the closest match to these sequences as being ' $C a$. Liberibacter asiaticus' strains gxpsy and psy62. The complete sequences of the serralysin-like genes from LsoB haplotype (1,479 bp, 'Ca. L. solanacearum' strain ZC1) and 'Ca. L. asiaticus' strain gxpsy have 493 and 497 amino acids, respectively. In contrast, the serralysin-like genes from the LsoA haplotype (1,983 bp, predicted from ' $C a$. L. solanacearum' strain HenneA) and ' $\mathrm{Ca}$. L. asiaticus' strain psy62 are predicted to encode much larger proteins, having 661 and 665 amino acids, respectively (Fig. 1A). In both ' $C a$. Liberibacter' spp., the T1SS genes are located upstream of the serralysin-like gene (Fig. 1B). Similarly, the T1SS genes for all four strains encode homologs for an ATPase (PrtD), a membrane fusion protein (HylD), and a $\mathrm{C}_{4}$-dicarboxylate transporter (DctA). Because of errors in the genome annotation of LsoA strain HenneA, we observed that the T1SS genes (ATPase, PrtD, and HylD) are erroneously broken (Fig. 1B). The serralysin-like proteins of ' $\mathrm{Ca}$. Liberibacter' spp. were compared with authentic serralysin protein sequences from $S$. marcescens and $S$. liquefaciens

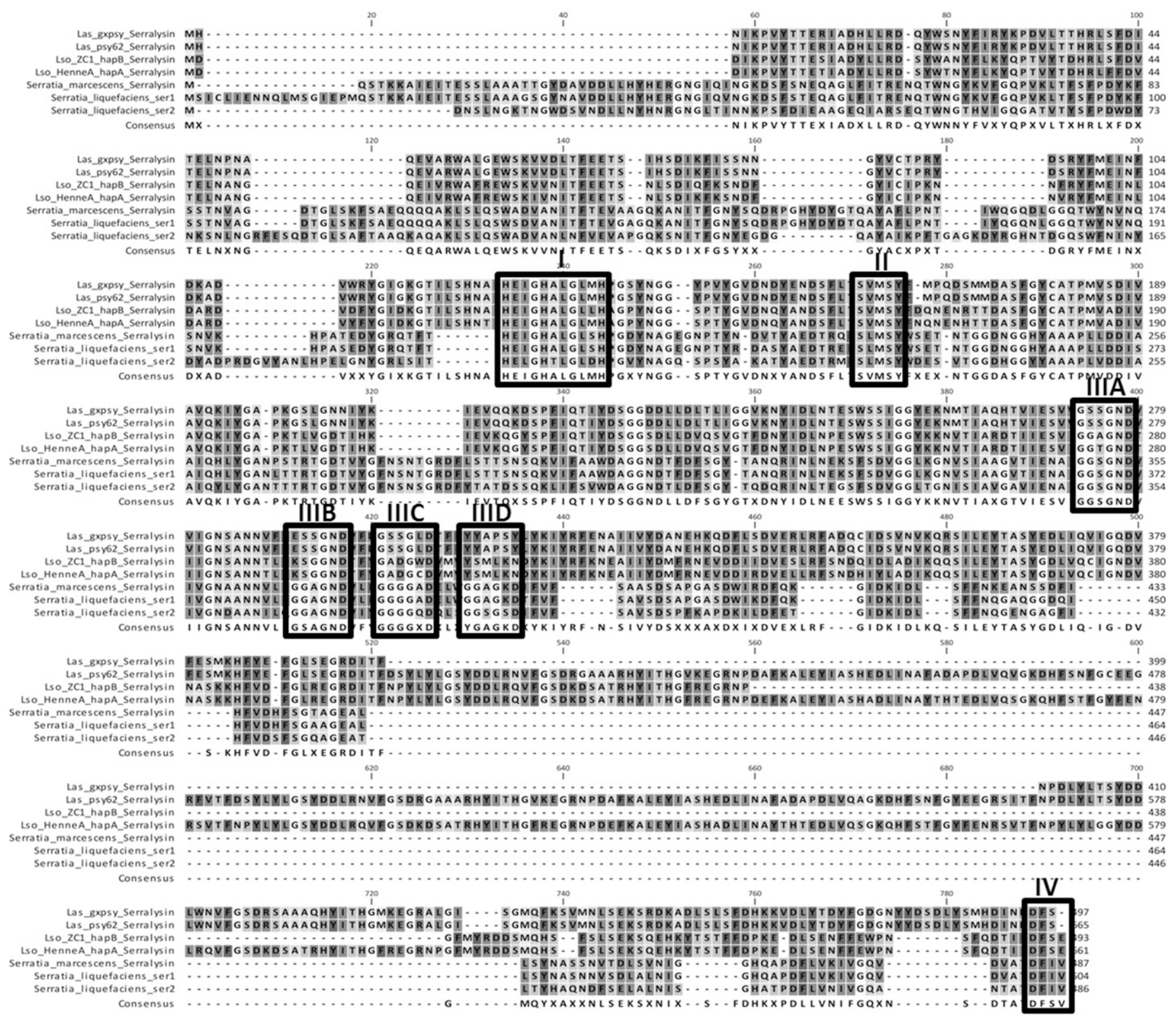

Fig. 2. Alignments of multiple serralysin family protein sequences from Serratia and 'Candidatus Liberibacter' spp. Conserved motifs associated with metalloprotease activity are shown in the dark boxes, including sequences encoding (I) a zinc-binding motif (HEXXHXUGUXH), (II) a Met-turn (SXMXY) motif, (IIIA-D) glycine-rich repeats (GGXGXD), and (IV) an ABC exporter motif (DXXX). 
FK01 (Kaibara et al. 2012). Conserved motifs for serralysins, including the zinc-binding domain (HEXXHXUGUXH), the Metturn sequence (SXMXY), the glycine-rich calcium-binding repeat sequences (GGXGXD), and the ABC exporter (DXXX) sequence (Fig. 2) all were identified in the predicted serralysin-like metalloproteases from both ' $\mathrm{Ca}$. L. solanacearum' haplotypes and both ' $\mathrm{Ca}$. L. asiaticus' strains. The authentic serralysin genes from both Serratia spp. contained four glycine-rich calcium-binding repeat sequences (designated IIIA, B, C, and D) (Fig. 2). In contrast, both ' $\mathrm{Ca}$. L. solanacearum' strains had only one repeat (position IIIA) (Fig. 2); the sequence of the binding repeat was (GGAGND) and (GGTGND) for the ' $C a$. L. solanacearum' strains $\mathrm{ZC1}$ and HenneA, respectively.

Expression of a ' $\mathrm{Ca}$. L. solanacearum' serralysin-like gene in tomato. To assess the expression of the ' $\mathrm{Ca}$. L. solanacearum' serralysin-like gene in planta, tomato leaves infected with the LsoB haplotype were collected at weeks 1, 2, 3, 4, and 6 following infestation with insects, time points before visible disease symptoms were apparent. qPCR analysis of gene expression with normalization of $\mathrm{Ct}$ values to the recA housekeeping gene showed that, at weeks $2,3,4$, and 6 , the expression of the ' $C a$. L. solanacearum' serralysin-like gene was 2.1- to 3.4-fold higher than at week 1, indicating that gene expression stays high as the disease progresses $\left(2^{-\Delta \Delta \mathrm{Ct}}\right.$ values were $2.1 \pm 0.4,2.4 \pm 0.6,2.9 \pm 0.6$, and $3.4 \pm 2.7$ at weeks $2,3,4$, and 6 , respectively, compared with week 1). In contrast, no amplification of the serralysin gene was observed in tomato plants infested with insects without ' $\mathrm{Ca}$. L. solanacearum'.
Functional studies of the ' $\mathrm{Ca}$. L. solanacearum' serralysin-like proteins. The complete sequences of the serralysin-like gene from the LsoA and LsoB haplotypes were cloned separately into a broadhost-range expression vector pBBR1-MCS4. As a control, the complete sequence of the $S$. liquefaciens serralysin ser 2 gene $(1,979 \mathrm{bp})$ was cloned into pBBR1-MCS4. Plasmids were transformed separately into the serralysin-deficient, serl/ser 2 double mutant $S$. liquefaciens FK04 and E. coli DE3 to test for protease activity. Because pBBR1-MCS4 is a low-copy plasmid, we were not able to clearly see the bands on an SDS-PAGE gel, including the positive control. The insertions were moved to pET14b, which is a high-copy expression plasmid; then, the protein sizes of the serralysin-like genes from the LsoA and LsoB haplotypes (approximately 75 and $56 \mathrm{kDa}$, respectively) were visible on SDS-PAGE gels, confirming the production of proteins at the predicted sizes (Supplementary Fig. S2).

On skim milk agar medium, neither of the ' $\mathrm{Ca}$. L. solanacearum' serralysin-like proteins expressed in S. liquefaciens FK04 exhibited enzymatic activity (Fig. 3A). In contrast, protease activity for authentic serralysin produced by $S$. liquefaciens FK01 or the double mutant $S$. liquefaciens FK04 with the ser 2 gene showed clear zones extending a few millimeters from the edge of the colony. In the casein zymography assay, surrogate cell culture supernatants and pellets were assayed for clear bands of proteolytic activity against a dark background (Fig. 3B). The S. liquefaciens serralysin (ser2) gene completely restored protease activity to the $S$. liquefaciens

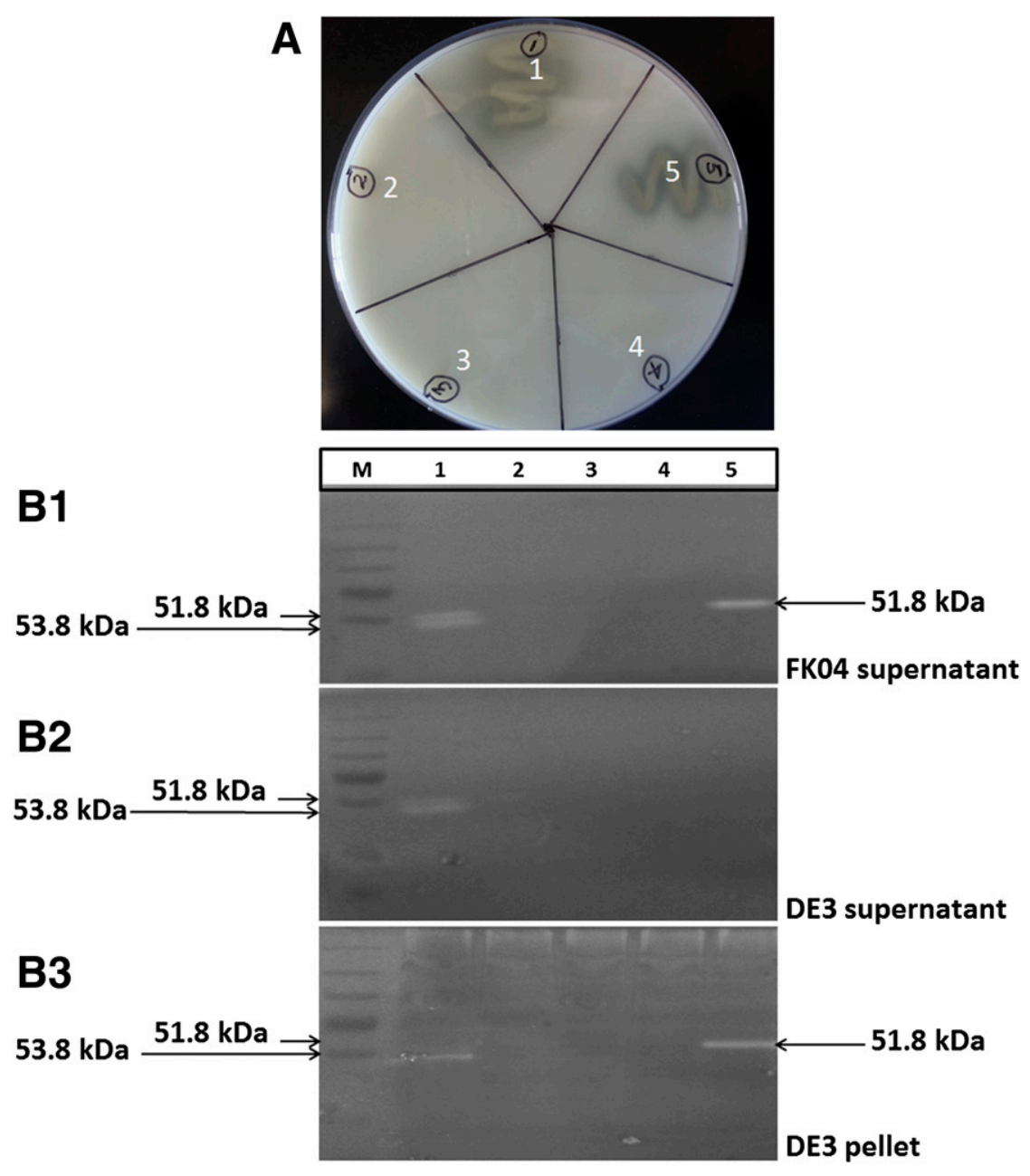

Fig. 3. Proteolytic activity on skim milk agar and casein zymography. A, The skim milk agar plate was incubated at $28^{\circ} \mathrm{C}$. Section 1 , FK01; 2 , FK04 double mutant; 3, FK04/pBBR1 LsoB ser; 4, FK04/pBBR1 LsoA ser; and 5, FK04/pBBR1 S1 ser2. B, Casein zymography for proteolytic activity. B1, Serratia liquefaciens FK04 supernatant: Lane M, protein marker; lane 1, FK01; lane 2, FK04; lane 3, FK04/pBBR1 LsoB ser; lane 4, FK04/pBBR1 LsoA ser; and lane 5, FK04/pBBR1 S1 ser2. B2, Escherichia coli DE3 supernatant and B3, pellet: Lane M, protein marker; lane 1, FK01; lane 2, FK04; lane 3, DE3/pBBR1 LsoB ser; lane 4, DE3/pBBR1 LsoA ser; and lane 5, DE3/pBBR1 S1 ser2. 
FK04 serralysin-deficient mutant (FK04/pBBR1 Sl ser2), whereas neither of the ' $\mathrm{Ca}$. L. solanacearum' serralysin-like genes restored protease activity in this mutant (FK04/pBBR1 LsoB ser or FK04/ pBBR1 LsoA ser) (Fig. 3B1). Expression of ' $\mathrm{Ca}$. L. solanacearum' serralysin-like genes in the nonserralysin-producing surrogate E. coli DE3 (DE3/pBBR1 LsoB ser and DE3/pBBR1 LsoA ser) also failed to convey protease activity (Fig. 3B2 and B3). Unexpectedly, there was no protease activity detected in the supernatant of E. coli harboring the pBBR Sl ser 2 construct (Fig. 3B2), whereas proteolytic activity was detected in the cell pellet (Fig. 3B3). Apparently, the $S$. liquefaciens serralysin was not secreted by E. coli DE3. Incompatibility between the signal peptide and secretion system for the two species also may be responsible for unsuccessful secretion of the ' $\mathrm{Ca}$. L. solanacearum' serralysin-like proteins expressed in either E. coli DE3 or S. liquefaciens FK04.

Functional studies of chimeric and truncated ' $C a$. $\mathrm{L}$. solanacearum' serralysin-like proteins. To account for the potential secretion incompatibility, we constructed chimeric sequences. For chimeric sequences, the entire region encoding the serralysin signature sequences encoding the putative catalytic domains from ' $C a$. L. solanacearum' were retained. This included the entire 3' region of the ' $\mathrm{Ca}$. L. solanacearum' gene encoding the zinc-binding domain, the Met-turn sequence, and the glycine-rich repeat. This ' $C a$. L. solanacearum' sequence was joined with the $5^{\prime}$ region of the $S$. liquefaciens FK01 sequence encoding the $\mathrm{N}$ terminal end of the serralysin protein thought to be involved in secretion. This process resulted in four different chimeras produced using combinations of the $5^{\prime}$ sequences from $S$. liquefaciens ser 1 and ser 2 and the $3^{\prime}$ sequences from the serralysin-like genes of the LsoA and LsoB haplotypes. The chimeric Serratia- 'Ca. L. solanacearum' serralysin-like protein was expressed in S. liquefaciens FK04 and the cell culture supernatants and pellets were assayed on skim milk agar and on a casein zymogram. None of the four chimeric serralysin-like gene-constructs (FK04/pBBR1 chimeric serl-LsoB, FK04/pBBR1 chimeric ser1-LsoA, FK04/pBBR1 chimeric ser2LsoB, and FK04/pBBR1 chimeric ser2-LsoA) restored protease activity to the serralysin-deficient mutant FK04 (data not shown). In contrast, serralysin protease activity was restored to the FK04 mutant by complementation with pBBR1-ser2 carrying the intact serralysin gene from $S$. liquefaciens.

As an alternative approach to chimeric sequences, we also constructed a truncated LsoB serralysin-like gene that carried only the three motifs associated with putative proteolytic activity in serralysin (i.e., excluding 300-bp 5' sequences that may not be involved in catalytic activity). As mentioned, the entire LsoA serralysin-like gene and LsoB serralysin-like gene and the truncated LsoB serralysin-like gene construct were cloned separately into the high-copy expression vector pET14b that generates a His-tagged protein for assays of proteolytic activity (zymogram and cholorometric assays). These plasmids were introduced into the E. coli DE3 surrogate. We focused on LsoB for the high expression vector study because it is typically the more virulent pathogen in our assays with potato (unpublished). As predicted, the complete LsoB serralysinlike genes resulted in the expression of proteins in E. coli DE3 of $56 \mathrm{kDa}$ (Fig. 4A1), as visualized on SDS-PAGE gels; the truncated construct produced a protein of $44 \mathrm{kDa}$ (Fig. 4A2). Neither of the intact proteins showed proteolytic activity in a zymogram assay (not shown). Neither the purified intact LsoB protein nor the truncated LsoB protein showed proteolytic activity in the colorimetric assay (Fig. 4B).

\section{DISCUSSION}

The importance of T1SS as the primary mechanism for secretion of ' $\mathrm{Ca}$. L. solanacearum' products involved in pathogen-microbe interactions was suggested by Lin et al. (2011) based on the absence of both T3SS and T2SS in the ' $\mathrm{Ca}$. L. solanacearum' genome. The finding that ' $\mathrm{C} a$. L. solanacearum' encodes a putative serralysin- like gene (CKC_02265) clustered together with a predicted T1SS locus identified the ' $C a$. L. solanacearum' serralysin-like gene as a highly interesting candidate virulence factor. Serralysin-like proteins are a family of metalloprotease produced by a wide range of microorganisms (Dahler et al. 1990; Maeda and Morihara 1995). Some of these extracellular metalloproteases have been either demonstrated or suggested to play critical roles in bacterial virulence to plants (Castro and Fontes, 2005). For example, Erwinia chrysanthemi produces a metalloprotease (PrtA) involved in virulence to modify or degrade host plant cell proteins during soft rot infection (Charkowski 2006; Dahler et al. 1990).

The in silico alignment of the predicted amino acid sequences for the ' $\mathrm{Ca}$. L. solanacearum' serralysin-like proteins with the predicted amino acid sequences of other serralysin family proteins showed a conserved zinc-binding motif, a Met-turn motif, a single glycine-rich calcium-binding repeat sequence, and an $\mathrm{ABC}$ exporter signal motif. These conserved motifs suggested that the 'Ca. L. solanacearum' serralysin-like protein was a member of the serralysin family of metalloproteases (Bode et al. 1993). The number of glycine-rich calcium-binding sequence repeats varies among bacterial strains along with the size of the protein (Basu and Apte 2008; Baumann 2011; Linhartová et al. 2010). The serralysin protein sequences from Serratia spp. have four glycine-rich calcium-binding repeats regions (Fig. 2), whereas both ' $C a$. L. solanacearum' haplotype sequences have only one (IIIA) (Fig. 2).

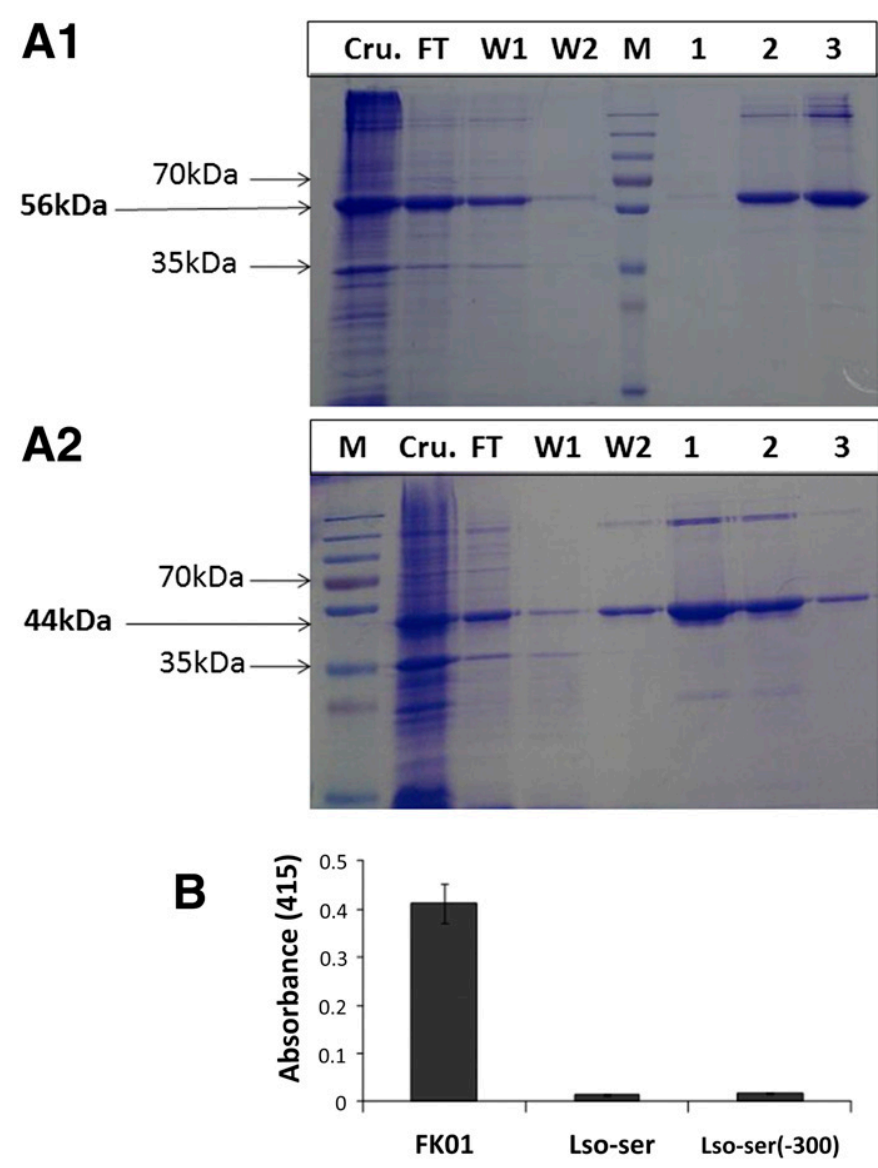

Fig. 4. Recombinant serralysin-like protein purity assayed by sodium dodecyl sulfate polyacrylamide gel electrophoresis and protease activity assayed by a colorimetric assay. A1, Purified LsoB serralysin-like proteins from construct pET14b LsoB ser and A2, purified truncated (5' deletion, $-300 \mathrm{bp}$ ) protein from construct pET14b LsoB ser-300. Lane M, protein marker (10 to 250 kDa); lane Cru., crude; lane FT, flow through; lane W1, wash 1; lane W2, wash 2; lane 1, elution fraction 1; lane 2, elution fraction 2; and lane 3, elution fraction 3. B, Colorimetric assay for protease activity from the culture supernatant of wild-type Serratia liquefaciens FK01, purified protein from construct pET14b LsoB ser, and purified protein from construct pET14b LsoB ser-300. 
Similarly, the two ' $\mathrm{Ca}$. L. asiaticus' sequences also appear to have only one GGXGXD sequence (IIIA) (Fig. 2) but neither sequence matched the consensus sequence perfectly, both having GSXGXD sequences. (Delepelaire 2004) reported that the three amino acids following the consensus repeat sequence also are important, and may form a short $\beta$-strand associated with the calcium-binding region (Baumann 2011). The three amino acids following the IIIA repeats in the serralysin sequences and the ' $\mathrm{Ca}$. L. asiaticus' sequences are all hydrophobic isoleucine or valine amino acids; two of three amino acids in the ' $\mathrm{Ca}$. L. solanacearum' sequences are hydrophobic. This pattern is less conserved following the other repeats. A characteristic of serralysin and its homologs is the binding of multiple calcium ions to the repeats-in-toxin (RTX) domain having these glycine-rich repeats. The binding of calcium ions is believed to facilitate the folding of serralysin and stabilizes the protein (Baumann 1994; Baumann et al. 1993). Our analysis suggests that, although the ' $\mathrm{Ca}$. L. solanacearum' serralysin-like gene encodes a protein with many of the putative catalytic domains, it lacks a prominent RTX domain rich in calcium-binding repeat sequences, which may have functional significance in terms of folding, catalytic activity, or both.

To investigate whether the ' $\mathrm{Ca}$. L. solanacearum' serralysin-like protein has functional protease activity, we used the $S$. liquefaciens FK01 mutant system (Kaibara et al. 2012) and Escherichia coli DE3 as expression surrogates. Our results reveal the importance of surrogate for demonstrating protease activity. The ' $\mathrm{Ca}$. L. solanacearum' serralysin-like genes did not restore protease activity to the serralysin-deficient S. liquefaciens FK04 mutant or convey activity to $E$. coli. In contrast, the $S$. liquefaciens serralysin (ser2) gene completely restored protease activity in the serralysindeficient S. liquefaciens FK04 mutant. However, proteolytic activity was detected only in the cell pellet (not the supernatant) of E. coli harboring the pBBR Sl ser 2 construct, suggesting that the $S$. liquefaciens serralysin was not secreted by E. coli DE3. Incompatibility between the signal peptide and secretion system appears to be important for exoprotease activity even for an authentic serralysin, which indicated that choice of surrogate is important for demonstrating exoprotease activity. We hypothesized that failure to separate the ' $C a$. L. solanacearum' serralysin-like proteins from the cell pellet when expressed in either $S$. liquefaciens or E. coli could have accounted for the lack of proteolytic activity in the supernatant.

To account for the potential secretion incompatibility, we constructed chimeric sequences having the $5^{\prime}$ sequence (potentially encoding factors related to secretion) from Serratia ser 1 or ser 2 and the $3^{\prime}$ sequence (potentially encoding catalytic activity) from LsoA or LsoB. However, none of the chimeric serralysin-like proteins demonstrated protease activity in the $S$. liquefaciens cell pellet or supernatant fractions. When the complete LsoB serralysin-like gene and the truncated gene having just the putative catalytic domain were expressed using a high-expression plasmid and purified from $E$. coli DE3, no proteolytic activity was observed for either purified protein, despite strong expression and yield in the pellet fractions. Although we obtained good separation of the protein from other cellular components, protein folding and stabilization could still have been an issue for activity in this experiment.

Although the ' $\mathrm{Ca}$. L. solanacearum' serralysin-like protein was produced at sufficiently high levels in both $E$. coli and S. liquefaciens for visualization on gels, the lack of proteolytic activity combined with the lack of a prominent RTX domain suggests several hypotheses: (i) the gene does not encode a functional metalloprotease; (ii) the protein does not fold properly on its own, may require other components to aid in folding, or cannot fold properly in these surrogates; (iii) the protein is missing some other requirement for protease activity (e.g., an additional component, chaperone, or cofactor); or (iv) the expression or activity levels are somehow below our technological capacity for detection. Our finding that the ' $\mathrm{Ca}$. L. solanacearum' serralysin-like gene expression increases 2.1- to 3.4-fold from the time insects are removed to 2, 3, 4, and 6 weeks after infection suggests a possible role for this protein in pathogenicity or survival in the plant host. Interestingly, the expression of the ' $\mathrm{Ca}$. L. asiaticus' serralysin-like was observed to be 5.5-fold higher in planta compared with within an Asian citrus psyllid, suggesting a more important role for the protein in plant-microbe compared with insect-microbe interactions (Yan et al. 2013). Although observations using our surrogate system indicate that the 'Ca. L. solanacearum' serralysin-like gene does not encode a functional serralysin-like metalloprotease, we hope our studies will provide useful insight for future work using other surrogates or assays. Nevertheless, the pattern of gene expression in planta suggests further work is needed to define the role of this gene in plant-pathogen interactions.

\section{ACKNOWLEDGMENTS}

We thank O. Huot, Texas A\&M University, for providing the infected psyllid and infected plant RNA samples; K. Iiyama, Kyushu University, Japan for providing S. liquefaciens FK01, FK02, FK03, and FK04; and M. Kovach, Baldwin Wallace University, Ohio for providing plasmid pBBR1 MCS-4.

\section{LITERATURE CITED}

Basu, B., and Apte, S. K. 2008. A novel serralysin metalloprotease from Deinococcus radiodurans. Biochim. Biophys. Acta 1784:1256-1264.

Baumann, U. 1994. Crystal structure of the $50 \mathrm{kDa}$ metallo protease from Serratia marcescens. J. Mol. Biol. 242:244-251.

Baumann, U. 2011. Serralysin. Encyclopedia of Inorganic and Bioinorganic Chemistry. John Wiley \& Sons, New Jersey.

Baumann, U., Wu, S., Flaherty, K. M., and McKay, D. B. 1993. Threedimensional structure of the alkaline protease of Pseudomonas aeruginosa: A two-domain protein with a calcium binding parallel beta roll motif. EMBO J. 12:3357-3364.

Bode, W., Gomis-Ruth, F. X., and Stockler, W. 1993. Astacins, serralysins, snake venom and matrix metalloproteinases exhibit identical zinc-binding environments (HEXXHXXGXXH and Met-turn) and topologies and should be grouped into a common family, the 'metzincins'. FEBS Lett. 331: 134-140.

Castro, M. S., and Fontes, W. 2005. Plant defense and antimicrobial peptides. Protein Pept. Lett. 12:11-16.

Charkowski, A. O. 2006. The soft rot Erwinia. Pages 423-505 in: PlantAssociated Bacteria. S. S. Gnanamanickam, eds. Springer, Dordrecht, The Netherlands.

Cong, Q., Kinch, L. N., Kim, B. H., and Grishin, N. V. 2012. Predictive sequence analysis of the Candidatus Liberibacter asiaticus proteome. PLoS One 7:e41071.

Dahler, G. S., Barras, F., and Keen, N. T. 1990. Cloning of genes encoding extracellular metalloproteases from Erwinia chrysanthemi EC16. J. Bacteriol. 172:5803-5815.

Delepelaire, P. 2004. Type I secretion in gram-negative bacteria. Biochim. Biophys. Acta 1694:149-161.

Diaz-Montano, J., Vindiola, B. G., Drew, N., Novy, R. G., Miller, J. C., and Trumble, J. T. 2014. Resistance of selected potato genotypes to the potato psyllid (Hemiptera: Triozidae). Am. J. Potato Res. 91:363-367.

Gottwald, T. R. 2010. Current epidemiological understanding of citrus Huanglongbing. Annu. Rev. Phytopathol. 48:119-139.

Haapalainen, M. 2014. Biology and epidemics of Candidatus Liberibacter species, psyllid-transmitted plant-pathogenic bacteria. Ann. Appl. Biol. 165:172-198.

Ishii, K., Adachi, T., Hamamoto, H., and Sekimizu, K. 2014. Serratia marcescens suppresses host cellular immunity via the production of an adhesion-inhibitory factor against immunosurveillance cells. J. Biol. Chem. 289:5876-5888.

Kaibara, F., Iiyama, K., Chieda, Y., Lee, J. M., Kusakabe, T., Yasunaga-Aoki, C., and Shimizu, S. 2012. Construction of serralysin-like metalloproteasedeficient mutants of Serratia liquefaciens and their virulence in the silkworm, Bombyx mori. J. Insect Biotechnol. Sericol. 81:55-61.

Kovach, M. E., Elzer, P. H., Hill, D. S., Robertson, G. T., Farris, M. A., Roop, R. M., and Peterson, K. M. 1995. Four new derivatives of the broad-hostrange cloning vector pBBR1MCS, carrying different antibiotic-resistance cassettes. Gene 166:175-176.

Lévy, J., Ravindran, A., Gross, D., Tamborindeguy, C., and Pierson, E. 2011. Translocation of 'Candidatus Liberibacter solanacearum', the Zebra Chip pathogen, in potato and tomato. Phytopathology 101: 1285-1291. 
Lévy, J. G., Scheuring, D. C., Koym, J. W., Henne, D. C., Tamborindeguy, C., Pierson, E., and Miller, J. C., Jr. 2015. Investigations on putative zebra chip tolerant potato selections. Am. J. Potato Res. 92:417-425.

Liefting, L. W., Weir, B. S., Pennycook, S. R., and Clover, G. R. 2009. 'Candidatus Liberibacter solanacearum', associated with plants in the family Solanaceae. Int. J. Syst. Evol. Microbiol. 59:2274-2276.

Lin, H., Lou, B., Glynn, J. M., Doddapaneni, H., Civerolo, E. L., Chen, C., Duan, Y., Zhou, L., and Vahling, C. M. 2011. The complete genome sequence of 'Candidatus Liberibacter solanacearum', the bacterium associated with potato zebra chip disease. PLoS One 6:e19135.

Linhartová, I., Bumba, L., Mašin, J., Basler, M., Osička, R., Kamanová, J., Procházková, K., Adkins, I., Hejnová-Holubová, J., Sadílková, L., Morová, J., and Šebo, P. 2010. RTX proteins: A highly diverse family secreted by a common mechanism. FEMS Microbiol. Rev. 34:1076-1112.

Maeda, H. 1996. Role of microbial proteases in pathogenesis. Microbiol. Immunol. 40:685-699.

Maeda, H., and Morihara, K. 1995. Serralysin and related bacterial proteinases. Methods Enzymol. 248:395-413.

Miyata, K., Tomoda, K., and Isono, M. 1971. Serratia protease. 3. Characteristics of enzyme as metalloenzyme. Agric. Biol. Chem. 35:460-467.

Munyaneza, J. E., Crosslin, J. M., and Upton, J. E. 2007. Association of Bactericera cockerelli (Homoptera: Psyllidae) with "zebra chip," a new potato disease in southwestern United States and Mexico. J. Econ. Entomol. 100:656-663.

Munyaneza, J. E., Fisher, T. W., Sengoda, V. G., Garczynski, S. F., Nissinen, A., and Lemmetty, A. 2010. Association of "Candidatus Liberibacter solanacearum" with the psyllid, Trioza apicalis (Hemiptera: Triozidae) in Europe. J. Econ. Entomol. 103:1060-1070.

Nachappa, P., Levy, J., Pierson, E., and Tamborindeguy, C. 2011. Diversity of endosymbionts in the potato psyllid, Bactericera cockerelli (Hemiptera: Triozidae), vector of zebra chip disease of potato. Curr. Microbiol. 62:1510-1520.
Ravindran, A., Levy, J., Pierson, E., and Gross, D. C. 2011. Development of primers for improved PCR detection of the potato zebra chip pathogen, 'Candidatus Liberibacter solanacearum'. Plant Dis. 95:1542-1546.

Secades, P., and Guijarro, J. A. 1999. Purification and characterization of an extracellular protease from the fish pathogen Yersinia ruckeri and effect of culture conditions on production. Appl. Environ. Microbiol. 65: 3969-3975.

Secor, G. A., Rivera, V. V., Abad, J. A., Lee, I. M., Clover, G. R. G., Liefting, L. W., Li, X., and De Boer, S. H. 2009. Association of 'Candidatus Liberibacter solanacearum' with zebra chip disease of potato established by graft and psyllid transmission, electron microscopy, and PCR. Plant Dis. 93:574-583.

Thompson, J. D., Gibson, J., Plewniak, F., Jeanmougin, F., and Higgins, D. G. 1997. The CLUSTAL_X windows interface: Flexible strategies for multiple sequences alignment aided by quality analysis tools. Nucleic Acids Res. 25: 4876-4882.

Wang, N., and Trivedi, P. 2013. Citrus huanglongbing: A newly relevant disease presents unprecedented challenges. Phytopathology 103:652-665.

Wen, A., Mallik, I., Alvarado, V. Y., Pasche, J. S., Wang, X., Li, W., Levy, L., Lin, H., Scholthof, H. B., Mirkov, T. E., Rush, C. M., and Gudmestad, N. C. 2009. Detection, distribution, and genetic variability of 'Candidatus Liberibacter' species sssociated with zebra complex disease of potato in North America. Plant Dis. 93:1102-1115.

Wen, A. M., Johnson, C., and Gudmestad, N. C. 2013. Development of a PCR Assay for the rapid detection and differentiation of 'Candidatus Liberibacter solanacearum' haplotypes and their spatiotemporal distribution in the United States. Am. J. Potato Res. 90:229-236.

Yan, Q., Sreedharan, A., Wei, S., Wang, J., Pelz-Stelinski, K., Folimonova, S., and Wang, N. 2013. Global gene expression changes in Candidatus Liberibacter asiaticus during the transmission in distinct hosts between plant and insect. Mol. Plant Pathol. 14:391-404. 